\title{
ON SOLUBILITY OF GROUPS WITH BOUNDED CENTRALIZER CHAINS
}

\author{
E. I. KHUKHRO \\ Sobolev Institute of Mathematics, Novosibirsk 630090, Russia \\ e-mail:khukhro@yahoo.co.uk
}

(Received 4 August 2007; accepted 25 September 2008)

\begin{abstract}
The $c$-dimension of a group is the maximum length of a chain of nested centralizers. It is proved that a periodic locally soluble group of finite $c$-dimension $k$ is soluble of derived length bounded in terms of $k$, and the rank of its quotient by the Hirsch-Plotkin radical is bounded in terms of $k$. Corollary: a pseudo-(finite soluble) group of finite $c$-dimension $k$ is soluble of derived length bounded in terms of $k$.
\end{abstract}

2000 AMS Classification. Primary: 20D10; Secondary: 03C20, 20D45, 20F16, $20 \mathrm{~F} 22$.

1. Introduction. Groups with the minimal condition for centralizers arise naturally in several areas of group theory. (It is easy to see that the ascending and descending chain conditions for centralizers are equivalent: $C_{G}(X)<C_{G}(Y) \Leftrightarrow$ $C_{G}\left(C_{G}(X)\right)>C_{G}\left(C_{G}(Y)\right)$.) In model theory, this condition proved to be useful in the study of stable groups [14]. Several interesting properties of groups with the minimal condition for centralizers were proved in $[\mathbf{2}-\mathbf{4}, \mathbf{6}, \mathbf{9}, \mathbf{1 5}]$.

If there is a uniform bound for the length of chains of centralizers, then it is convenient to use the following terminology proposed by A. Myasnikov and P. Shumyatsky in [12] (where this stronger condition was used in the study of discriminating groups).

Definition. The $c$-dimension of a group $G$ is the maximum length of a nested chain of centralizers of subsets in $G$.

The same notion is also known as the height of the lattice of centralizers. There are several important classes of groups of finite $c$-dimension: abelian groups, stable groups (in model theory), torsion-free hyperbolic groups, linear groups over fields (or over finite direct products of fields), which include free groups, finitely generated nilpotent groups, polycyclic groups, finitely generated metabelian groups, finitely generated abelian-by-nilpotent groups. In addition, the class of groups of finite $c$-dimension is closed under universal equivalence and under taking subgroups and finite direct products. (Of course, some of these classes have the property in question due to certain theorems by various authors; see $[7,12]$.)

In the present paper we, in particular, obtain a bound for the derived length of a finite soluble group in terms of its $c$-dimension. This result obviously extends to periodic locally soluble groups as well as to so-called pseudo-(finite soluble) groups. Earlier, such a bound was known for locally nilpotent groups [3]. We denote by $F(G)$ the Hirsch-Plotkin radical of a group $G$, which is the largest normal locally nilpotent 
subgroup of $G$, and by $F_{2}(G)$ the inverse image in $G$ of $F(F / F(G))$. We abbreviate 'bounded in terms of $k$ ' to ' $k$-bounded'.

THEOREM 1. If a periodic locally soluble group $G$ has finite c-dimension $k$, then

(a) $G$ is soluble of $k$-bounded derived length;

(b) the quotient $G / F(G)$ by the Hirsch-Plotkin radical has k-bounded rank; and therefore

(c) the quotient $G / F_{2}(G)$ by the second Hirsch-Plotkin subgroup has an abelian subgroup of finite $k$-bounded index.

Earlier R. Bryant and B. Hartley [4] proved that a periodic locally soluble group with the minimal condition for centralizers

(1) is soluble,

(2) has a nilpotent normal subgroup with quotient of finite rank, and

(3) has a nilpotent-by-abelian subgroup of finite index.

Theorem 1(a) strengthens part (1) under the stronger hypothesis of finite $c$-dimension $k$ by giving a bound in terms of $k$ to the derived length of the group. One might expect that in the case of finite $c$-dimension $k$ there must be similarly stronger versions of parts (2) and (3) by analogy with linear groups. However, we produce examples showing that there may not exist a nilpotent-by-abelian subgroup of finite $k$-bounded index. Another example shows that there may not exist a nilpotent normal subgroup of $k$-bounded class with quotient of finite rank. (The examples in [4] also highlight the difference between linear groups and groups with the minimal condition for centralizers.) It remains unclear if there must exist a nilpotent subgroup with quotient of finite $k$-bounded rank.

It would be interesting to combine our theorem with the classification of finite simple groups to yield results in the more general case of locally finite (not necessarily locally soluble) groups of finite $c$-dimension. Note that O. Kegel [9] proved that a locally finite group of finite exponent with the minimal condition on centralizers is nilpotent-by-finite.

Conjecture (A. V. Borovik). Let $G$ be a locally finite group of finite $c$-dimension $k$. Let $S$ be the full inverse image of the 'generalized Fitting subgroup' $F^{*}(G / F(G))$, which is equal to the product of $F(G / F(G))$ and all the quasi-simple subnormal subgroups of $G / F(G)$. Then

(1) the number of non-abelian simple composition factors of $G$ is finite and $k$-bounded;

(2) $G / S$ has an abelian subgroup of finite $k$-bounded index.

In view of the importance of the $c$-dimension in model theory, it seems worth stating a corollary for pseudo-(finite soluble) groups. (In general, a group is pseudofinite if it is elementarily equivalent to an ultraproduct of finite groups.)

THEOREM 2. Suppose that a group $G$ is elementarily equivalent to an ultraproduct of finite soluble groups. If $G$ has finite $c$-dimension $k$, then $G$ is soluble of $k$-bounded derived length.

Note that D. Macpherson and K. Tent [11] proved that a stable pseudofinite group has a definable normal soluble subgroup of finite index. Other results on pseudofinite groups include J. Wilson's characterization of simple pseudofinite groups [16]. One 
may expect certain combinations of these results with our Theorems 1 and 2 to yield further consequences for arbitrary pseudofinite groups of finite $c$-dimension.

It is possible to give an explicit estimate for the function of $k$ in the theorems, but we do not write it out.

2. Proofs. The proof of Theorem 1 uses the Hall-Higman theorems and the following theorem of J. Thompson [13] on automorphisms of soluble groups. Let $h(X)$ denote the Fitting height of a finite soluble group $X$.

THOMPSON'S THEOREM. Suppose that a finite soluble group G has an automorphism $\alpha$ of prime order coprime to the order of the group: $(|\alpha|,|G|)=1$. Then $h(G) \leqslant 5 h\left(C_{G}(\alpha)\right)$.

We shall freely use the simple fact that the $c$-dimension of any proper centralizer $C_{G}(X) \neq G$ is strictly smaller than the $c$-dimension of $G$. It is also clear that the $c$-dimension of a subgroup does not exceed that of the group.

The first step in the proof of Theorem 1(a) is the case of nilpotent groups, where a sharp bound for the derived length can be found in the paper of R. Bryant [3] (who attributes the result to T. Yen); we reproduce the proof here for the benefit of the reader.

LEMMA 1. If a nilpotent group $N$ has finite c-dimension $k$, then the derived length of $N$ is at most $k$.

Proof. Induction on $k$. If $k=1$, then the group is abelian and there is nothing to prove. For $k>1$, choose any non-central element $g$ in the second centre: $g \in \zeta_{2}(N) \backslash$ $\zeta_{1}(N)$. Then $N \neq C_{N}(g) \geqslant[N, N]$. Since the $c$-dimension of $C_{N}(g)$ is at most $k-1$, by the induction hypothesis $C_{N}(g)$ has derived length at most $k-1$, and therefore so does $[N, N]$.

Proof of Theorem 1(a). It is obviously sufficient to consider finitely generated and therefore finite subgroups of $G$, so we simply assume that $G$ is finite. We proceed by induction on $k$. The case $k=1$ is trivial, so we assume that $k>1$.

By Lemma 1 the derived length of all Sylow subgroups of $G$ is at most $k$. Therefore it is sufficient to bound the Fitting height of $G$.

By the Hall-Higman theorems it follows from Lemma 1 that the $p$-length of $G$ is at most $k$ for each prime $p$. (Originally, this was proved by P. Hall and G. Higman [8] for odd primes, then T. Berger and F. Gross [1] obtained the bound $2 k-2$ for $p=2$ and finally the case of $p=2$ was finished by E. Bryukhanova [5] with the best possible bound $k$.)

Let $h=h(G)$ be the Fitting height of $G$; we may of course assume that $h>1$. We now fix a prime divisor $p$ of $\left|G / F_{h-1}(G)\right|$, the last non-trivial Fitting quotient of $G$. Let $H$ be a Hall $p^{\prime}$-subgroup of $F_{h-1}(G)$. Clearly, it is now sufficient to bound the Fitting height of $H$.

\section{Lemma 2. The Fitting height of $H$ is $k$-bounded.}

Proof. Since the Hall $p^{\prime}$-subgroups of $F_{h-1}(G)$ are conjugate in $F_{h-1}(G)$, by an analogue of Frattini's argument we have $G=N_{G}(H) F_{h-1}(G)$. Therefore we can choose a $p$-element $a$ in $N_{G}(H) \backslash F_{h-1}(G)$. If $a$ centralizes $H$, then we complete the proof by the induction hypothesis of Theorem 1 applied to $C_{G}(a) \geqslant H$. Indeed, $C_{G}(a)$ has $c$-dimension at most $k-1$, since $C_{G}(a) \neq G$ for $a \notin F_{h-1}(G)$. 
Thus, we can assume that $\left|N_{G}(H) / C_{G}(H)\right|$ is divisible by $p$. Let $\beta$ be the automorphism of order $p$ in Aut $H$ induced by conjugation by some element $b \in N_{G}(H)$. Then $C_{H}(\beta)=C_{H}(b)$ has $c$-dimension at most $k-1$. By the induction hypothesis the derived length of $C_{H}(\beta)$ is bounded by a number $f=f(k-1)$. In particular, the Fitting height of $C_{H}(\beta)$ is bounded by the same number. Now, by Thompson's theorem, the Fitting height of $H$ is at most $5 f$.

Since the $p$-length of $G$ is at most $k$, Lemma 2 implies that the Fitting height of $G$ is $k$-bounded. Since the derived length of every Sylow $q$-subgroup, for any prime $q$, is at most $k$ by Lemma 1 , this completes the proof of Theorem 1(a).

For part (b) we shall need the following lemma.

LEMMA 3. If an elementary abelian p-group $E$ of order $p^{n}$ acts faithfully on a finite nilpotent $p^{\prime}$-group $Q$, then there is a series of subgroups $E=E_{0}>E_{1}>E_{2}>\cdots>E_{n}=1$ such that all the inclusions $C_{Q}\left(E_{0}\right)<C_{Q}\left(E_{1}\right)<\cdots<C_{Q}\left(E_{n}\right)$ are strict.

Proof. Induction on $n$. If $n=1$, then $C_{Q}(E) \neq C_{Q}(1)=Q$, since the action is faithful.

Let $n>1$. First suppose that $[Z(Q), E] \neq 1$. Since the action is coprime, we have $C_{[Z(Q), E]}(E)=1$. Let $V$ be a minimal non-trivial $E$-invariant subgroup of $[Z(Q), E]$. Then $V$ is an elementary $q$-group, which can be regarded as an irreducible $\mathbb{F}_{q} E$ module. Therefore, $E / C_{E}(V)$ is cyclic, so that $\left|C_{E}(V)\right|=p^{n-1}$. By the induction hypothesis, $C_{E}(V)$ has a series $C_{E}(V)=E_{1}>\cdots>E_{n}=1$ such that all the inclusions $C_{Q}\left(E_{1}\right)<\cdots<C_{Q}\left(E_{n}\right)$ are strict. Since $E$ acts non-trivially on $V \leqslant C_{Q}\left(E_{1}\right)$, we also have $C_{Q}(E) \neq C_{Q}\left(E_{1}\right)$, which completes the proof in the case $[Z(Q), E] \neq 1$.

In the general case, choose a maximal normal subgroup $N \leqslant C_{Q}(E)$. By the properties of coprime action, $E$ acts faithfully on $Q / N$ and non-trivially on $Z(Q / N)$ by the maximal choice of $N$. By the above, there is a series of subgroups $E=E_{0}>E_{1}>$ $E_{2} \cdots>E_{n}=1$ such that all the inclusions $C_{Q / N}\left(E_{0}\right)<C_{Q / N}\left(E_{1}\right)<\cdots<C_{Q / N}\left(E_{n}\right)$ are strict. Since each centralizer $C_{Q / N}\left(E_{i}\right)$ is the image of $C_{Q}\left(E_{i}\right)$, it follows that all the inclusions $C_{Q}\left(E_{0}\right)<C_{Q}\left(E_{1}\right)<\cdots<C_{Q}\left(E_{n}\right)$ are also strict.

Proof of Theorem 1(b). By the inverse limit argument we can again assume that $G$ is a finite soluble group.

It is known [10] that the rank of $G / F(G)$ is at most 1 plus the maximum of the ranks of Sylow subgroups of $G / F(G)$. Let $P$ be a Sylow $p$-subgroup of $G / F(G)$; we can also regard $P$ as a Sylow $p$-subgroup of $G / O_{p}(G)$. Let $Q=F\left(G / O_{p}(G)\right)$; then $Q$ is a nilpotent $p^{\prime}$-subgroup. We can regard $P$ as a Sylow $p$-subgroup of $\left(G / O_{p}(G)\right) / Q$, which acts faithfully on $Q$. It is sufficient to bound the rank of a maximal abelian normal subgroup $A$ of $P$. This is due to the well-known fact that the rank of a $p$-group $P / A$ of automorphisms of an abelian $p$-group $A$ is bounded in terms of the rank of $A$.

In turn, the rank of $A$ is equal to the rank of an elementary abelian subgroup $E \leqslant A$. Let $H$ be a Hall $p^{\prime}$-subgroup of the full inverse image of $Q$ in $G$, so that $O_{p}(G) H / O_{p}(G)=Q$. By the generalized Frattini argument, $O_{p}(G) N_{G}(H)=G$. Hence the semidirect product $Q E$ is isomorphic to a subgroup of $N_{G}(H)$. Therefore, $Q E$ has $c$-dimension at most $k$. By Lemma 3 it follows that the rank of $E$ is at most $k$.

Proof of Theorem 1(c). We can again assume that $G$ is a finite soluble group. Let $R=G / F(G)$ and let $r$ be the rank of $R$. For every prime $p$ the quotient $R / O_{p^{\prime}, p}(R)$ is a linear group of dimension $\leqslant r$ in characteristic $p$, since it acts faithfully on the Frattini quotient of $O_{p^{\prime}, p}(R) / O_{p^{\prime}}(R)$. By the Lie-Zassenhaus-Kolchin-Mal'cev 
theorem, $R / O_{p^{\prime}, p}(R)$ has a triangularizable normal subgroup of $r$-bounded index. Since the unitriangular part of $R / O_{p^{\prime}, p}(R)$ is trivial, $R / O_{p^{\prime}, p}(R)$ has an abelian subgroup of $r$-bounded index. Hence, by Remak's theorem, $R / F(R)=R / \bigcap_{p} O_{p^{\prime}, p}(R)$ has an abelian subgroup of $r$-bounded exponent. Combined with the bound for the rank of $G / F(G)$ given by part (b), this gives a bound for the index of $F_{2}(G)$ in terms of $k$.

Proof of Theorem 2. We can obviously assume that $G=\prod_{i \in I} G_{i} / \mathcal{U}$, where the $G_{i}$ are finite soluble groups and $\mathcal{U}$ is a non-principal ultrafilter on the set of indices $I$. The property of a group to have $c$-dimension at most $k$ can be written by a universal formula of the first-order language of group theory. Therefore, since $G$ has this property, the set $J$ of indices $i$ such that $G_{i}$ has $c$-dimension at most $k$ belongs to $\mathcal{U}$. By Theorem 1 these groups $G_{i}$ for $i \in J$ are soluble of derived length at most $f(k)$, where $f(k)$ depends only on $k$. Since $J \in \mathcal{U}$, the ultraproduct $G$ is also soluble of derived length at most $f(k)$.

\section{Examples.}

EXAMPLE 1. Choose large primes $p, q$ such that $q-1$ is divisible by $p$ and consider the semidirect product $Q P$ of a normal cyclic group $Q$ of order $q$ and a cyclic group $P \leqslant$ Aut $Q$ of order $p$, where $C_{Q}(P)=1$. Let $U$ be a vector space over a finite field of characteristic $r \neq p, q$ admitting a fixed-point-free linear action of $Q$. For simplicity we can choose $r$ so that $r-1$ is divisible by $q$, and let $Q$ act on a one-dimensional space $U$ over $\mathbb{F}_{r}$ as an automorphism group of order $q$ of a cyclic group of order $r$, so that $C_{U}(Q)=0$. Let $V$ be the space of the representation of $Q P$ induced from the representation of $Q$ on $U$. Let $G=V Q P$ be the natural semidirect product, where $V$ also denotes the additive group of the vector space $V$. Then, for example, $\left|C_{V}(P)\right|=r$ and $C_{V}(Q)=0$. It is easy to see that the $c$-dimension of $G$ is 3 , while the index of a nilpotent-by-abelian subgroup is at least $p$.

EXAMPLE 2. Let $p$ be a prime. Consider a free group $G$ in the variety $\mathfrak{A}^{2} \cap \mathfrak{N}_{p-1} \cap \mathfrak{B}_{p}$ of metabelian nilpotent groups of class $p-1$ and of exponent $p$ on free generators $x_{1}, x_{2}, \ldots$ We claim that $C_{G}(x)=\langle x\rangle Z(G)$ for any $x \in G \backslash[G, G]$. This obviously also implies that $C_{G}(v)=[G, G]$ for any $v \in[G, G] \backslash Z(G)$. Then the only chains of centralizers are $G>[G, G]>Z(G)>1$ and $G>\langle x\rangle Z(G)>Z(G)>1$, so the $c$-dimension of $G$ is 3 .

Indeed, it is well known that the group $[G, G]$ can be regarded as a vector space over $\mathbb{F}_{p}$ with a basis formed by all the simple basic commutators of weight $\leqslant p-1$ in the free generators

$$
\left[x_{i_{1}}, x_{i_{2}}, x_{i_{3}}, \ldots, x_{i_{n}}\right], \quad i_{1}>i_{2} \leqslant i_{3} \leqslant \ldots \leqslant i_{n}, \quad n \leqslant p-1 .
$$

Let $x \in G \backslash[G, G]$; clearly, $C_{G}(x) \leqslant\langle x\rangle[G, G]$. Let $[c, x]=1$ for $c \in[G, G]$; we need to show that $c \in \gamma_{p-1}=Z(G)$. Let $c, x \in\left\langle x_{1}, \ldots, x_{m}\right\rangle$. It is known that $x$ can be included in a system of free generators of $G$. Thus we can assume that $x=x_{m}$. But $\left[\varkappa, x_{m}\right]$ is again a simple basic commutator for any simple basic commutator $\varkappa$ in $x_{1}, \ldots, x_{m}$. Hence $\left[c, x_{m}\right]=1$ only if $c$ is a linear combination of basic commutators of maximal weight $p-1$, which all belong to $Z(G)$.

Thus, the $c$-dimension of $G$ is 3 , but any normal subgroup with quotient of finite rank has nilpotency class exactly $p-1$. By considering finitely generated subgroups of $G$ we also obtain finite $p$-groups of $c$-dimension 3 without a bound for the rank of the quotient by a subgroup of nilpotency class $\leqslant p-2$. 
ACKNOWLEDGEMENTS. The author thanks Alexandre Borovik and John Wilson for stimulating discussions.

\section{REFERENCES}

1. T. R. Berger and F. Gross, 2-length and the derived length of a Sylow 2-subgroup, Proc. Lond. Math. Soc. 34(3) (1977), 520-534.

2. V. V. Bludov, On locally nilpotent groups with the minimality condition for centralizers, Algebra Logika 37 (1998), 270-278; English transl. in Algebra and Logic 37 (1998), 151-156. $371-383$

3. R. M. Bryant, Groups with minimal condition on centralizers, J. Algebra 60 (1979),

4. R. M. Bryant and B. Hartley, Periodic locally soluble groups with the minimal condition on centralizers, J. Algebra 61 (1979), 328-334.

5. E. G. Bryukhanova, Connection between the 2-length and the derived length of a Sylow 2-subgroup of a finite solvable group, Mat. Zametki 29 (1981), 161-170; English transl. in Math. Notes 29 (1981), 85-90.

6. J. Derakhshan and F. O. Wagner, Nilpotency in groups with chain conditions, Quart. J. Math. Oxford 48 (1997), 453-468.

7. A. J. Duncan, I. V. Kazachkov and V. N. Remeslennikov, Centraliser dimension and universal classes of groups, Siberian Electronic Math. Rep. 3 (2006), 197-215; http://semr.math.nsc.ru.

8. P. Hall and G. Higman, On the $p$-length of $p$-soluble groups and reduction theorems for Burnside's problem, Proc. Lond. Math. Soc. 6(3) (1956), 1-42.

9. O. Kegel, Four lectures on Sylow theory in locally finite groups, in Group Theory Proc. Int. Conf. Singapore, 1987, Walter de Gruyter, Amsterdam, 1989, pp. 3-27.

10. L. G. Kovács, On finite soluble groups, Math. Z. 103 (1968), 37-39.

11. D. Macpherson and K. Tent, Stable pseudofinite groups, J. Algebra 312 (2007), 550-561.

12. A. Myasnikov and P. Shumyatsky, Discriminating groups and $c$-dimension, J. Group Theory 7 (2004), 135-142.

13. J. Thompson, Automorphisms of solvable groups, J. Algebra 1 (1964), 259-267.

14. F. O. Wagner, Stable groups, mostly of finite exponent, Notre Dame J. Formal Logic 34 (1993) 183-192.

15. F. O. Wagner, Nilpotency in groups with the minimal condition on centralizers, J. Algebra 217 (1999), 448-460.

16. J. S. Wilson, On pseudofinite simple groups, J. Lond. Math. Soc. 51(2) (1995), 471-490. 